\title{
Transport mechanism in lightly doped hydrogenated microcrystalline silicon thin films
}

\author{
A. Dussan ${ }^{\text {a) }}$ \\ INTEC (UNL-CONICET), Güemes 3450, 3000 Santa Fe, Argentina \\ R. H. Buitrago \\ INTEC (UNL-CONICET), Güemes 3450, 3000 Santa Fe, Argentina and FIQ (UNL), Santiago del Estero \\ 2829, 3000 Santa Fe, Argentina
}

(Received 22 April 2004; accepted 16 November 2004; published online 28 January 2005)

\begin{abstract}
Boron-doped microcrystalline silicon films have been deposited in a plasma-enhanced chemical vapor deposition system using silane diluted in hydrogen, and diborane $\left(\mathrm{B}_{2} \mathrm{H}_{6}\right)$ as a dopant gas. The temperature dependence of the dark conductivity has been measured from 120 to $420 \mathrm{~K}$ in all samples. In the high-temperature range above room temperature, the carrier transport is found to be thermally activated, with a single activation energy that changes with the $\mathrm{B}_{2} \mathrm{H}_{6}$ compensation degree. In the low-temperature range (300-120 K), variable range hopping (VRH) was established as a predominant electronic transport mechanism for all samples, with the exception of the sample with a diborane concentration of $12.5 \mathrm{ppm}$. A model for Mott's VRH, referred to as the "diffusional model," which yields a relation between the conductivity and the localized density of gap states, is presented. Using classical equations from the percolation theory and the diffusional model, the density of states near the Fermi level, as well as the hopping parameters, are calculated. A correlation between the hopping parameters for both models is deduced. A numerical factor that improves the value of each parameter is calculated. () 2005 American Institute of Physics.
\end{abstract}

[DOI: 10.1063/1.1848193]

\section{INTRODUCTION}

In recent years, hydrogenated microcrystalline silicon $(\mu \mathrm{c}-\mathrm{Si}: \mathrm{H})$ has played an important role in the development of electronic devices such as solar cells, transistors and gas detectors. ${ }^{1,2}$ The study and understanding of the transport mechanisms in this material has become a basic research topic. Numerous papers have investigated ${ }^{3,4}$ correlations among electronic, optical, and micro structural properties with electronic transport models. However, no general theory providing an explanation for all the observed behaviors has been presented to date.

In the range of temperatures well below zero degrees centigrade, it has been reported ${ }^{5}$ that $\mu \mathrm{c}-\mathrm{Si}: \mathrm{H}$ dark conductivity $\left(\sigma_{d k}\right)$ does not follow a thermally activated model, which is the common case above room temperature. Different models have been proposed, but how charges respond to an electric field is still unclear.

In this paper, results on dark conductivity of microcrystalline silicon thin films lightly doped with Boron, $\mu \mathrm{c}-$ $\mathrm{Si}: \mathrm{H}:(\mathrm{B})$, measured over a temperature range of $100-420 \mathrm{~K}$ are shown. Well-known theories for disordered materials, like variable range hopping (VRH) ${ }^{6}$ were applied. It was found that the Golden Rule of fitting $\ln \left(\sigma_{d k}\right) v s T^{-1 / 4}$ to identify hopping mechanisms is a necessary but not a sufficient condition. In the case of $\mu \mathrm{c}-\mathrm{Si}: \mathrm{H}:(\mathrm{B})$, some corrections are required to find reasonable hopping parameters.

According to Mott's equation ${ }^{6}$ for the three-dimensional

${ }^{\text {a)} E l e c t r o n i c ~ m a i l: ~ a d u s s a n c @ i n t e c . u n l . e d u . a r ~}$ case, if the density of states (DOS) is constant in a $k_{B} T$ energy range around the Fermi level, the dark conductivity as a function of temperature is given by

$$
\sigma=\sigma_{0}^{*} \exp \left[-\left(\frac{T_{0}}{T}\right)^{1 / 4}\right]
$$

where $T$ is the absolute temperature, $\sigma_{0}^{*}$ and $T_{0}$ are constants which depend on the material.

Many disordered or amorphous semiconductors and metal alloys follow this equation. ${ }^{7,8}$ Recently, this behavior has been reported not only in the range of low temperatures and constant DOS, as it was originally predicted by Mott and David, ${ }^{9}$ but also at high temperatures and for materials with a nonconstant DOS around the Fermi level. ${ }^{10,11}$ Different kinds of functions can be proposed for the DOS in the gap and the band tails of these semiconductors. This point, however, will not be considered in this paper.

All the lightly doped microcrystalline silicon samples follow Mott's equation at low temperatures. Following the classical percolation theory, the characteristic hopping parameters, and the distance between states and energy differences were calculated. Results were in disagreement with the expected range of values. Therefore, we developed a "diffusional model" considering the Einstein relation, conductivity, diffusion coefficient, and hopping probability, which can be correlated with the percolation model equations to provide a method for the adoption of the correct numerical coefficients of both models. 


\section{FUNDAMENTAL THEORIES FOR VRH}

\section{A. Diffusional model}

Considering a VRH regimen, it can be proposed that the carriers move in a diffusive way and have a diffusion coefficient $D_{\text {hop }}$ given by ${ }^{12}$

$$
D_{\text {hop }}=\frac{1}{6} R^{2} p_{\text {hop }},
$$

where $R$ is the range or average hopping distance and $p_{\text {hop }}$ is the hopping transition probability between two states separated a distance $R$ from each other. The transition probability can be written in general as ${ }^{12}$

$$
p_{\text {hop }}=\nu_{\text {ph }} \exp \left(-2 \alpha R-W / k_{B} T\right),
$$

where $\nu_{\mathrm{ph}}$ is the phonon frequency associated with the hopping processes; $\alpha^{-1}$ is the wavelength overlapping parameter; and $k_{B}$ is the Boltzmann's constant. Therefore, $\exp (-2 \alpha R)$ is the tunneling factor given by the overlapping of wave functions, and $\exp \left(-W / K_{B} T\right)$ is the Boltzmann's factor, which correlates with energy $W$ between the initial and final hopping states.

If it is assumed that the density of states is constant in a range of some $k_{B} T$ around the Fermi energy, and the latter is within the localized states, then for the three-dimensional case, the number of states per unit volume and energy $\left(N_{F}\right)$, separated by a hopping distance $R$, is related to the energy difference between hopping states by:

$$
W=\frac{3}{4 \pi R^{3} N_{F}} .
$$

Parameter $W$ is also denominated as the "hopping activation energy," and is one of the most important parameters in identifying the hopping mechanism. This parameter will be used to compare different models.

Using Eq. (4) into (3), and evaluating the maximum of the function, we obtain the optimum distance $R$ which allows us to get the higher rate of hopping ${ }^{13}$

$$
R=\left[\frac{9}{8 \pi \alpha N_{F} k_{B} T}\right]^{1 / 4} \text {. }
$$

The Einstein relation allows the calculating of the dark conductivity, $\sigma_{d k}$, in terms of the diffusion coefficient and the density of states according to ${ }^{13,14}$

$$
\sigma_{d k}=e^{2} D_{\text {hop }} N_{F},
$$

where $e$ is the electronic charge, $D_{\text {hop }}$ is the diffusion coefficient, and $N_{F}$ is the density of states near the Fermi level.

Combining Eqs. (2)-(6), the following equation for the dark conductivity was found:

$$
\sigma=\frac{1}{6} e^{2} R^{2} N_{F} \nu_{\mathrm{ph}} \exp \left[-\left(\frac{18.1 \alpha^{3} / N_{F} k_{B}}{T}\right)^{1 / 4}\right],
$$

which is similar to Mott's equation if we call

$$
\sigma_{0}^{*}=\frac{1}{6} e^{2} R^{2} N_{F} \nu_{\mathrm{ph}}
$$

and

$$
T_{0}=\frac{18.1 \alpha^{3}}{N_{F} k_{B}} .
$$

As dark conductivity and temperature are easily measured in thin film materials, from a plot of $\ln \left(\sigma_{d k}\right) \mathrm{vs} 1 / T^{1 / 4}$, the slope $T_{0}$ can be determined, and by choosing a reasonable value for $\alpha$, the density of states at the Fermi level can be estimated. Subsequently, through Eqs. (4) and (5) the hopping parameters $R$ and $W$ can be calculated. Thus, the diffusional model gives us a method to calculate the hopping characteristic parameters, where only the very well-known $\alpha$ parameter have to be assumed.

\section{B. Percolation theory}

The percolation theory has been widely used to study transport phenomena in semiconductors, in particular to calculate the hopping parameters of Mott's equation for the case of VRH. The essence of such an approach lies in the fact that the spheres of radius $R$, on sites along a most favored path, must join up to form a percolation channel through the material. As a result, the spheres would overlap, offering problems to scientists in the estimation of $R$. There are a number of approaches being reported as having the same mathematical expression but a different multiplying coefficient, which ranges from 1.78 to 2.48 . $^{9}$ On average, calculated values of $R$ are smaller than the ones estimated by other methods. As a consequence, when used in Eq. (4), the resulting values for the hopping energies are higher than the expected ones. The proposed formulas ${ }^{15,16}$ can be summarized as follows:

$$
T_{0}=\frac{C^{4} \alpha^{3}}{k_{B} N_{F}},
$$

where $C$ is a number in the range of 1.84-2.28 predicted for a constant density of states around the Fermi level. ${ }^{16}$ Comparing this to the value obtained for $T_{0}$ from the diffusional model, it was found that $C^{4}=18.1$, being $C=2.06$, which is clearly within the range of percolation values. It is important to notice that the same value for Mott's temperature is obtained from two different models.

For the rest of the characteristic VRH parameters, the classical equations reported are ${ }^{15,16}$

$$
\begin{aligned}
& W=k_{B}\left(T_{0} T^{3}\right)^{1 / 4}, \\
& R=\frac{3}{8} T_{0}^{1 / 4} T^{-1 / 4}\left(\frac{1}{\alpha}\right) .
\end{aligned}
$$

As both models allow us to calculate $R$ and $W$, there should be a correlation between them. By including Eqs. (8) and (10) into (4), and Eq. (8) into (5), we obtain the following new expressions after some mathematical operations:

$$
\begin{aligned}
& W^{*}=\frac{A}{C^{4}} k_{B}\left(T_{0} T^{3}\right)^{1 / 4}, \\
& R^{*}=\frac{B}{C} T_{0}^{1 / 4} T^{-1 / 4}\left(\frac{1}{\alpha}\right),
\end{aligned}
$$

where $A=(128 / 9 \pi)$ and $B=(9 / 8 \pi)$.

It is interesting to notice that the new equations [Eqs. (11) and (12)] maintain the same structure of the ones predicted by the percolation theory, having a pre-factor which is a function of parameter $C$. Recently, Godet et al. ${ }^{11}$ reported 


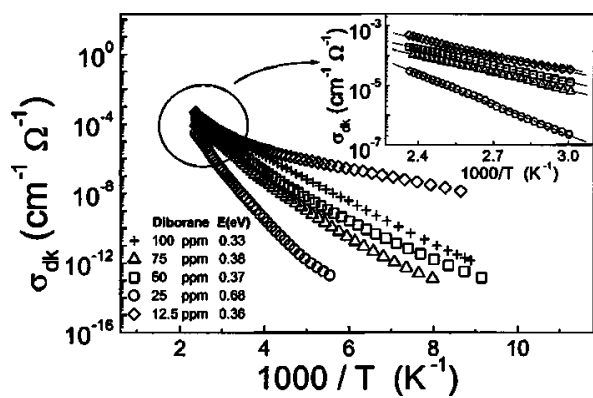

FIG. 1. Dark conductivity as a function of the inverse temperature for $\mu \mathrm{c}-\mathrm{Si}: \mathrm{H}$ samples with a diborane concentration between 12.5 and $100 \mathrm{ppm}$. The figure inset shows a lineal fitting for all samples in the high-temperature range. $E$ is the activation energy.

that $C^{4}$ could reach the value of 310 for materials having an exponential density of states, instead of the maximum value of 16 admitted by Mott's theory for a constant density of states. Therefore, these results indicate that the correlation between different models and the shape of the density of states depend on the value of $C$.

\section{EXPERIMENT}

The $\mu \mathrm{c}-\mathrm{Si}: \mathrm{H}:(\mathrm{B})$ samples were prepared in a capacitively coupled HF-PECVD reactor working at a frequency of $50 \mathrm{MHz}$. A description of the characteristics and the operational conditions of the reactor were reported elsewhere. ${ }^{10}$ Corning 7059 glass was used as a substrate, and the deposition temperature was $150{ }^{\circ} \mathrm{C}$. The reaction gas was a mixture of $94 \%$ hydrogen and $6 \%$ silane, containing diborane in the range of $0-100 \mathrm{ppm}$. The total flux and pressure were kept constant at $20 \mathrm{sccm}$ and $4.5 \times 10^{-1}$ Torr, respectively.

Conductivity measurements were carried out in a cryostat using thermally evaporated aluminum coplanar electrodes. They were $25 \mathrm{~cm}$ in length, interdigited, and separated $0.01 \mathrm{~cm}$ from each other. The electrical current was measured with a Keithley electrometer 617 connected to a computer. The samples were annealed at $420 \mathrm{~K}$ and then cooled down to $120 \mathrm{~K}$ at a constant rate of $1.5 \mathrm{~K} / \mathrm{min}$. The applied field was $1000 \mathrm{~V} / \mathrm{cm}$.

Modulated photocurrent measurements (MPC) were performed with the same contact configuration. A He-Ne laser was partially modulated ( $12 \%$ of its total intensity) by using an electro-optical modulator at $4 \mathrm{kHz}$. The dc generation rate was $2.7 \times 10^{17} \mathrm{~cm}^{-3} \mathrm{~s}^{-1}$ and the applied field was 4

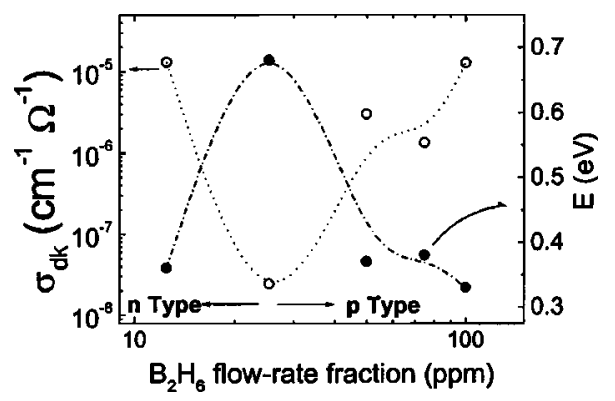

FIG. 2. Room temperature dark conductivity and activation energy for $\mu \mathrm{c}$-Si:H samples with different boron doping degrees. The lines are drawn to guide the eye.

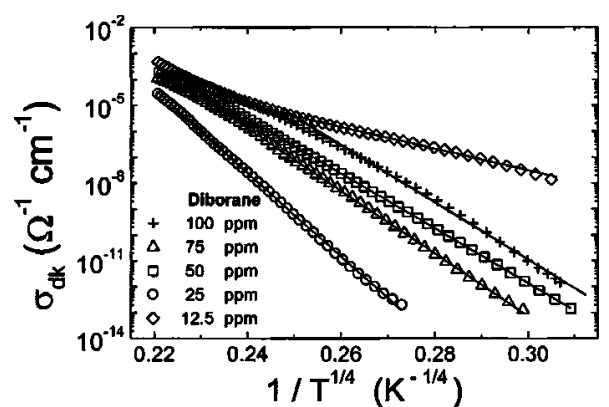

FIG. 3. Dark conductivity vs $T^{-1 / 4}$ for $\mu \mathrm{c}-\mathrm{Si}: \mathrm{H}$ samples lightly doped with $B$.

$\times 10^{4} \mathrm{~V} / \mathrm{cm}$. Phase lag and amplitude of the MPC signal were simultaneously detected with a lock-in amplifier.

\section{RESULTS AND DISCUSSION}

Figure 1 shows an Arrhenius plot of the dark conductivity of the lightly doped $\mu \mathrm{c}-\mathrm{Si}: \mathrm{H}:(\mathrm{B})$ samples. A linear behavior is only observed in the high-temperature region, as shown in the figure inset. In this temperature region (over $25^{\circ} \mathrm{C}$ ), the transport mechanism involves carriers activated directly from levels below the Fermi level to states at the bottom of the conduction (or valence) band. The activation energies $(E)$ indicate that the samples change from a $n$-type to a $p$-type semiconductor passing through a compensated $\mu \mathrm{c}-\mathrm{Si}: \mathrm{H}:(\mathrm{B})$ sample (doping around $25 \mathrm{ppm}$ ), as it is shown in Fig. 2. The intrinsic microcrystalline hydrogenated silicon $(\mu \mathrm{c}-\mathrm{Si}: \mathrm{H})$ is a $n$-type semiconductor. If a small amount of Boron is added, which acts as an impurity acceptor, it will push down the Fermi level from the conduction to the valence band, changing the microcrystalline material to $p$-type. Being the conductivity an exponential function of the activation energy, small changes in the Fermi level will result in large variations of $\sigma_{d k}$, as it can be seen in Fig. 2 .

Since the Arrhenius plot does not fit at low temperatures, with the exception of the sample doped with $12.5 \mathrm{ppm}$ of diborane, other possible transport mechanisms were sought. In Fig. 3, the variation of dark conductivity versus $1 / T^{1 / 4}$ is shown. At low temperatures, the lineal fitting is very good, giving the expected correlation of Eq. (1). Using Mott's equation, the slopes $T_{0}$ were determined (Table II). Therefore, based on the diffusional model for $T_{0}$ and the density of states measured in our laboratory by the method of modu-

TABLE I. Wavelength overlapping $\left(\alpha^{-1}\right)$ calculated from the diffusional model. Values of $N_{F}$ are experimental DOS data measured by the MPC method.

\begin{tabular}{|c|c|c|}
\hline $\begin{array}{c}\text { Sample } \\
\left(\mathrm{ppm} \mathrm{B} \mathrm{B}_{2} \mathrm{H}_{6}\right)\end{array}$ & $\begin{array}{c}N_{F} \\
\left(\mathrm{~cm}^{-3} \mathrm{eV}^{-1}\right)\end{array}$ & $\begin{array}{l}\alpha^{-1} \\
(\AA)\end{array}$ \\
\hline 12.5 & $1 \times 10^{16}$ & 61 \\
\hline 25 & $7.8 \times 10^{15}$ & 11 \\
\hline 50 & $1.01 \times 10^{16}$ & 18 \\
\hline 75 & $6.80 \times 10^{15}$ & 17 \\
\hline 100 & $5.04 \times 10^{16}$ & 10 \\
\hline
\end{tabular}


TABLE II. DOS values and hopping parameters $(T=150 \mathrm{~K})$ from the diffusional and percolation models.

\begin{tabular}{cccccccc}
\hline \hline & & & \multicolumn{2}{c}{ T. Percolation } & & \multicolumn{2}{c}{ M. Diffusional } \\
& & & & & & \\
Sample $\left(\mathrm{ppm} \mathrm{B}_{2} \mathrm{H}_{6}\right)$ & $T_{0}(\mathrm{~K})$ & $N_{F}\left(\mathrm{eV}^{-1} \mathrm{~cm}^{-3}\right)$ & $R(\mathrm{~cm})$ & $W(\mathrm{eV})$ & & $R(\mathrm{~cm})$ & $W(\mathrm{eV})$ \\
\hline 12.5 & $9.15 \times 10^{7}$ & $8.31 \times 10^{17}$ & $1.46 \times 10^{-6}$ & 0.36 & & $1.46 \times 10^{-6}$ & 0.09 \\
25 & $1.90 \times 10^{10}$ & $4 \times 10^{15}$ & $5.57 \times 10^{-6}$ & 1.37 & & $5.57 \times 10^{-6}$ & 0.34 \\
50 & $3.55 \times 10^{9}$ & $2.13 \times 10^{16}$ & $3.66 \times 10^{-6}$ & 0.9 & & $3.66 \times 10^{-6}$ & 0.22 \\
75 & $6.07 \times 10^{9}$ & $1.25 \times 10^{16}$ & $4.18 \times 10^{-6}$ & 1.03 & & $4.19 \times 10^{-6}$ & 0.25 \\
100 & $4.24 \times 10^{9}$ & $1.79 \times 10^{16}$ & $3.82 \times 10^{-6}$ & 0.94 & & $3.83 \times 10^{-6}$ & 0.23 \\
\hline \hline
\end{tabular}

lated photocurrent (MPC), ${ }^{17}$ the wave length overlapping parameter was determined. The results for $\alpha^{-1}$ and DOS are shown in Table I.

Table I shows that $\alpha^{-1}$ varies in the range of $10-18 \AA$, with the exception of the sample having a diborane concentration of $12.5 \mathrm{ppm}$, a fact that will be discussed later. The agreement between the $\alpha^{-1}$ values found here and the ones predicted by the percolation theory ${ }^{18}$ is worth noticing. Taking this into consideration, the mean value of $\alpha^{-1}$ equal to $14 \AA$ will be adopted for the next calculations.

Table II shows the results for the hopping parameters $R$ and $W$, calculated from the diffusional model [Eqs. (4) and (5)] and the percolation theory [Eqs. (9) and (10)] at a temperature of $150 \mathrm{~K}$. The density of states at the Fermi level, $N_{F}$, was calculated using Eq. (8) and the values of $C=2.06$ and $\alpha^{-1}=14 \AA$. These results present a fair agreement with the experimental values obtained by the MPC method, ${ }^{19}$ reported in Table I. The sample doped with $12.5 \mathrm{ppm}$ shows a discrepancy of one order of magnitude between the measured and calculated $N_{F}$ values. Besides, if it is taken into account that the $\alpha^{-1}$ values calculated in Table I are relatively high, it should be considered that the possibility that the transport mechanism for this sample is different from VRH, even when Mott's equation is satisfied. A nearest-neighbor hopping behavior was checked, since the results were negative. It is concluded that for this sample the mechanism may be a carrier transport controlled by thermoionic emission, ${ }^{20}$ as a reasonable value of $101 \mathrm{meV}$ was obtained for the potential barrier.

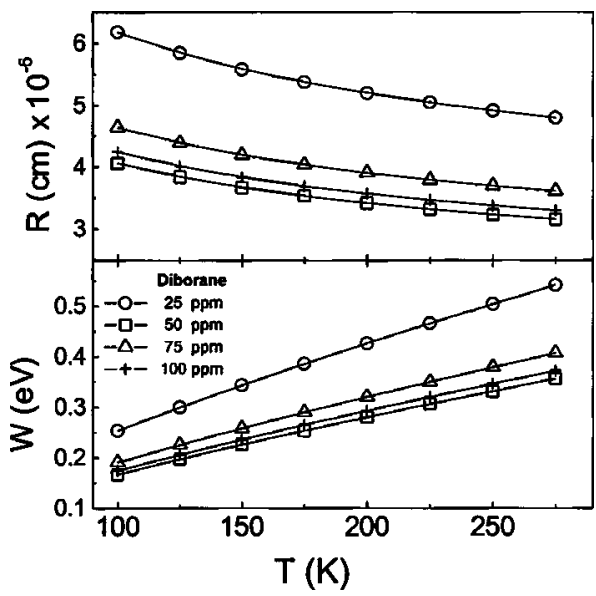

FIG. 4. Hopping parameters $R$ and $W$ as a function of temperature for $\mu \mathrm{c}-\mathrm{Si}: \mathrm{H}$ samples, according to the diffusional model equations.
Figure 4 shows the dependence of $R$ and $W$ on temperature. Both curves behave as expected by the VRH mechanism, i.e., when temperature decreases, $R$ increases and $W$ decreases.

The $R$ values obtained either from the diffusional or the percolation models are the same, but the hopping energies $W$ are quite different. The value predicted by the percolation method for $W$ gives a value equivalent to the gap of the material. This value is in contradiction with the basic initial assumption that the density of states was constant around the Fermi level, and that the jump of the carriers from one state to the other were only of the order of some $k_{B} T$. On the other hand, the initial assumptions are satisfied for the value of $W$ obtained from the diffusional model.

Recalculating $R^{*}$ and $W^{*}$ from Eqs. (11) and (12) using $C=2.06$, we again obtain the same values shown in Table II, corresponding to the diffusional model.

\section{CONCLUSIONS}

Dark conductivity measurements performed over a wide range of temperatures for lightly doped $\mu \mathrm{c}: \mathrm{Si}: \mathrm{H}: \mathrm{B}$ samples are reported in this paper. It was found that for temperatures higher than $25{ }^{\circ} \mathrm{C}$ the dominant mechanism is the thermal activation of carriers. The different activation energies found are explained in terms of boron doping.

In the low-temperature ranges, the main transport mechanism for the $\mu \mathrm{c}-\mathrm{Si}: \mathrm{H}: \mathrm{B}$ samples is the VRH, with the exception of the case doped with $12.5 \mathrm{ppm}$ of diborane. From the diffusional model formulas, and using experimental data for the density of states, parameters $C$ and $\alpha^{-1}$ were calculated. A good agreement was obtained with the predicted value from the percolation theory.

A correlation between diffusional and percolation model formulas for $R$ and $W$ was deduced. The resulting equations preserve the same mathematical structure of the percolation equations multiplied by a pre-factor, which is a function of parameter $C$. Introducing the $C$ value obtained from the diffusional model, the values obtained from the new equations for $R^{*}$ and $W^{*}$ are reasonable for a VRH model with a constant density of states around the Fermi level. Higher value of $C^{4}$ would indicate that new basic assumptions are necessary in addition to Mott's condition. 


\section{ACKNOWLEDGMENTS}

This work was supported by the Universidad Nacional del Litoral (CAI+D 2000, 6-1-84) and by ANPCyT (project PICT 12-06950).

${ }^{1}$ A. V. Shah, J. Meier, E. Vallet-Sauvain, N. Wyrsch, U. Kroll, C. Droz, and U. Graf, Sol. Energy Mater. Sol. Cells 78, 469 (2003).

${ }^{2}$ M. Kondo, Y. Nasumo, H. Mase, T. Wada, and A. Matsuda, J. Non-Cryst. Solids 299, 108 (2002).

${ }^{3}$ M. Goerlitzer, P. Torres, N. Beck, N. Wyrsch, H. Keppner, J. Pohl, and Shah, J. Non-Cryst. Solids 227, 996 (1998).

${ }^{4}$ M. F. Cerqueira, J. A. Ferreira, and G. J. Adriaenssens, Thin Solid Films 370, 128 (2000).

${ }^{5}$ P. Hapke, U. Backhausen, R. Carius, F. Figer, and S. Ray, Mater. Res. Soc. Symp. Proc. 420, 789 (1996).

${ }^{6}$ N. F. Mott, Philos. Mag. 19, 333 (1969).

${ }^{7}$ D. Adler and H. Fritzsche, Tetrahedrally-Bonded Amorphous Semiconductors (Plenum Press, New York and London, 1985).

${ }^{8}$ A. R. Long and L. Hansmann, Hopping and Related Phenomena, edited by H. Fritzsche and M. Pollak (Word Scientific, Singapore, 1990).

${ }^{9}$ N. F. Mott and R. A. Davis, Electronic Processes in Non-Crystalline Materials, 2nd ed. (Oxford University Press, Oxford, 1979).

${ }^{10}$ S. B. Concari, R. H. Buitrago, M. T. Gutierrez, and J. J. Gandia, J. Appl. Phys. 94, 2417 (2003).

${ }^{11}$ C. Godet, J. Non-Cryst. Solids 299, 333 (2002).

${ }^{12}$ R. A. Street, Hydrogenated Amorphous Silicon (Cambridge University Press, Cambridge, 1991).

${ }^{13}$ Amorphous Semiconductors: Topics in Applied Physics, edited by M. H. Brodsky (Springer, Berlin 1979), Vol. 36.

${ }^{14}$ G. Paasch, T. Lindner, and S. Scheinert, Synth. Met. 132, 97 (2002).

${ }^{15}$ S. B. Concari and R. H. Buitrago, J. Non-Cryst. Solids 338-340, 331 (2004).

${ }^{16} \mathrm{M}$. Thamilselvan, K. Premnazeer, D. Mangalaraj, and Sa. K. Narayandass, Physica B 337, 404 (2003).

${ }^{17}$ C. Longeaud and J. P. Kleider, Phys. Rev. B 45, 11672 (1992).

${ }^{18}$ R. M. Hill, Philos. Mag. 24, 1307 (1971).

${ }^{19}$ A. Dussan, R. R. Koropecki, R. Arce, J. A. Schmidt, and R. Buitrago, J. Non-Cryst. Solids 338-340, 430 (2004).

${ }^{20}$ A. Di Nocera, A. Mitiga, and A. Rubino, J. Appl. Phys. 78, 3955 (1995). 